\section{Strafverfahren gegen Frauenhändler}

Birgit Thoma

$S_{h}$ in chätzungen der UN gehen davon aus, dass in Westeuropa jährlich 500.000 Frauen "gehandelt« werden, davon 50.000 nach Deutschland. Eine ausdifferenzierte Statistik zu den verschiedenen Formen des Handels - ob zur sexuellen Ausbeutung, zur Ausbeutung der Arbeitskraft oder zur Eingehung der Ehe - existiert nicht. Lediglich die im Lagebild Menschenhandel des BKA erfassten Zahlen zum Prostitutionshandel sind deutlich angestiegen und haben sich seit 2001 fast verdoppelt. ${ }^{1}$ Die Frauen kommen überwiegend aus den Mittel- und osteuropäischen Staaten und hier vor allem aus Russland, Lettland, Litauen, Bulgarien und Rumänien. ${ }^{2}$ Auf der Seite der Täter handelt es sich zu 40 $\%$ um deutsche Staatsangehörige und der Anteil von weiblichen Tätern liegt derzeit bei $25 \%$. Auch die Phänomenologie des Frauenhandels hat sich in den letzten Jahren deutlich verändert: Der Organisationsgrad der Täter nimmt zu, die Opfer werden immer jünger und die Fälle in denen sie unter Drogen gesetzt, betäubt oder unter Gewaltanwendung entführt werden steigen. Auch konnte bisher die List der Täter - die falschen Versprechungen auf eine Arbeit im Gaststättengewerbe, als Haushaltshilfe, oder eine Heirat mit den Vermittlern (»Romeo-Effekt «) nicht adäquat erfasst werden. Die Täter mussten lediglich behaupteten, die Frauen wären freiwillig mitgegangen und hätten auch geahnt was ihnen droht. Der Tatnachweis war folglich schwierig und in der Konsequenz wurden bisher weniger als $10 \%$ der Täter verurteilt. Soviel zum status quo. Wie soll er durch die Neuregelung, einer (verspäteten) Reaktion auf internationale Vorgaben ${ }^{3}$ verändert werden?

\section{Menschenhandel als Freiheitsdelikt}

Geregelt ist Menschenhandel neuerdings im 18. Abschnitt, den Delikten gegen die persönliche Freiheit. Diese Umwertung erweitert zugleich den Schutz auf alle ArbeitnehmerInnen ${ }^{4}$ und damit auf Prostitutions-, Arbeits- und Heiratshandel. Eine - auf den ersten Blick - sachgerechte Verschiebung. Systematisch und nach Sinn und Zweck der Neuregelung kann es jetzt nur noch auf die Beeinflussung oder Beugung des Willens ankommen. Die Betroffenen verlassen die Sicherheit des eigenen (oder eines anderen) Wohnsitzlandes und setzen sich den Unsicherheiten aus, die mit dem Aufenthalt in einem anderen Land verbunden sind. Dafür ist es unerheblich, zu welchem Zweck Verletzte des Menschenhandels in das andere Land einreisen, ob sie etwa wissen oder auch nur vermuten, dass sie dort bspw. der Prostitution nachgehen sollen, und erst recht ist irrelevant, welche Tätigkeit sie zuvor ausgeübt haben, ob sie also beispielsweise bereits im Herkunftsland als Prostituierte gearbeitet haben oder nicht. Die Kehrseite der Medaille ist allerdings, dass die persönliche Freiheit ein disponibles Rechtsgut ist, die Einwilligung also rechtfertigt. Im folgenden ein kurzer Überblick über die Neuregelungen:

- Aktives »Einwirken « auf die Frauen im Sinne eines Zwangs oder von wahrheitswidrigen Versprechungen ist nicht mehr erforderlich, es reicht bereits der Versuch mit Frauen $z u$ handeln und sie zu überreden.

- Die Abstufungen in den einzelnen Tatbeständen sind erheblich und reichen von drei Monaten bis zu zehn Jahren.

- Ein Vermögensvorteil muss weder angestrebt noch bewiesen werden. Es genügt, wenn in die Prostitution vermittelt oder durch sexuelle Handlungen ausgebeutet wird (Peepshows, pornographischer Darstellungen, ggf. Heiratsschwindelei ${ }^{5}$ ).

- Das hohe Schutzalter von 21 Jahren wurde für die Prostitutionsvermittlung, Arbeitsausbeutung und ausbeutende sexuelle Handlungen beibehalten ${ }^{6}$.

- Kinderhandel ist grundsätzlich ein Verbrechen.

- Eine besonders unbefriedigende Gesetzeslücke wurde durch den neuen $\S 233$ a StGB geschlossen: Künftig sind auch Förderungshandlungen, also beihilfeartige Handlungen zum Menschenhandel (z.B. das Beherbergen oder Befördern) als eigenständige Taten erfasst.

- $§ 233$ b StGB ermöglicht es Führungsaufsicht anzuordnen. Außerdem wird die Gewinnabschöpfung durch die Gerichte erleichtert.

- Da die Fälle von Entführungen bisher nur unzureichend über § 239 StGB erfasst waren, wurde die Regelung des Menschenraubs nach $\S 234$ StGB geschaffen.

- Bei der Strafverfolgung wurde mit $\S 154$ c StPO die Möglichkeit geschaffen von einer Strafverfolgung abzusehen, wenn die Betroffene gegen ausländer- oder arbeitsrechtliche Vorschriften verstoßen hat. Dadurch soll die Bereitschaft zur Aussage gefördert werden.

\section{Anmerkungen: \\ Grad der Ausbeutung entscheidet über Freiheitsschutz}

Auch die neu gefassten Regelungen enthalten einige Unstimmigkeiten, die in der Praxis vorhersehbar zu Problemen führen werden. In § 232 Abs. 12 . Alternative wird lapidar auf »ausbeuterische Sexualkontakte " verwiesen, jedoch nicht definiert was genau darunter zu verstehen ist. Die Begründung verweist hier auf die Definition des $\S 180$ a Abs. 2 S. 2 StGB $^{7}$, d.h. künftig muss eine wirtschaftliche Ausbeutung ${ }^{8}$ nachgewiesen werden. Aber wie? - dies bleibt schleierhaft und wird die Praxis vor Beweisschwierigkeiten stellen. Bisher greift eine solche wirtschaftliche Ausbeutung nur für Fälle von vermarkteten pornografischen Darstellungen und für Peepshows, denn in beiden Fällen besteht ein krasses Missverhältnis zwischen Leistungen die die Frauen - wenn überhaupt - erhalten und den Gewinnen, die die Täter damit erzielen. Wie aber wird künftig ein krasses Missverhältnis in Fällen der Zwangsprostitution zu bewerten sein? Reicht es aus, dass die Täter behaupten, die Frauen hätten auch »marktgerechte " Gegenleistungen erhalten? Bisher genügen hier Schätzungen $^{9}$ und es wird davon ausgegangen, dass eine Ausbeutung vorliegt, wenn die Frauen mindestens die Hälfte ihrer Einnahmen abführen und von dem ihr verbleibenden Anteil wiederum die Hälfte abgeben und weiter die Tagesmiete bezahlen müssen. Die Folgen: erstens entscheiden die Preise des Marktes über die Tatbestandsmäßigkeit und zweitens liegt de facto die Beweislast bei den Frauen. Unzumutbar, denn meist können die Frauen nicht beweisen, wie viel der Täter an ihnen verdient hat, da sie keinen Einblick in die Verdienst-und Ausbeutungsspannen der Täter haben.

Systematisch verbietet sich eigentlich ein Rückgriff auf die Rechtsprechung zu $§ 180$ a Abs. 2 S. 2 StGB da m. E. dadurch die Grenzen zwischen Prostitution unter Zwangs- und Situationen der Freiwilligkeit verwischt und dadurch auch die Schutzgüter der einzelnen Regelungen nicht klar getrennt werden. Die Zwangssituation kann nicht an Kriterien des Prostitutionsgesetzes gemessen und dadurch »ökonomisch relativiert « werden. Daher ist bei der Bewertung der Ausbeutung nicht auf die wirtschaftliche, sondern vor allem die persönliche Ausbeutung durch den Eingriff in die Freiheitsrechte der betroffenen Frauen abzustellen.

\section{Schutzaltergrenzen}

Kinderhandel (14 Jahre) ist qualifiziert. Aber die Bundesregierung bleibt mit dieser Schutzaltergrenze bewusst (wie übrigens 2004 schon bei der Kinderpornografie) hinter den Vorgaben des EU-Rahmenbeschlusses zurück, der »Kinder« als 
Personen unter 18 Jahren definiert und generell für diese Gruppe verschärfende Qualifikationen vorsieht.

Ein weiteres Problem sind unklare und sich überschneidende Schutzaltergrenzen. In § 232 Abs. 12 . Alt. StGB sind es 21 Jahre, aber es ist nicht ersichtlich wovor Heranwachsende generell besonders geschützt werden sollen. Schieflagen ergeben sich dadurch zu $\S 180$ Abs. 1 und 2, 182 StGB - 16 und/oder 18 Jahre. Minderjährige unter 18 Jahren dürfen zwar nicht zu entgeltlichen sexuellen Handlungen mit Dritten bestimmt oder zu solchen Sexualkontakten vermittelt werden. Wenn jemand aber selbst Sexual-kontakte mit minderjährigen Prostituierten sucht, gilt nach § 182 StGB nur eine Schutzaltergrenze von 16 Jahren. Erst bei genauerer Betrachtung kann der sich zunächst aufdrängende Wertungswiderspruch relativiert werden, da $\S 182$ eine Freierbestrafung bedeutet und nicht das Schutzalter für Vermittlungen regelt. Daraus ergibt sich, dass §§ 180,180a Abs. 2 StGB differenzierter sind als § 232 Abs. 1 2. Alt. StGB. Sie sprechen für eine Wahlfreiheit ab 18 Jahren zu entscheiden, ob Prostitution der richtige Beruf ist. Das Vermittlungsverbot in $\S 232$ Abs. 12 . Alternative StGB ist daher problematisch. Die Schutzaltergrenze macht daher nur bei klaren Zwangssituationen Sinn. Da die Betroffenen nach den Hellfeld-Daten meist zwischen 18 und 24 Jahre alt sind, sollten die Anforderungen bei über 18 Jährigen höher angesetzt werden. Die Gesetzgebung scheint kein angemessenes Konzept gehabt zu haben.

\section{Sanktionsebene: Erweiterter Verfall}

Die Neuregelung sieht in $§ 233$ b StGB die Gewinnabschöpfung durch den erweiterten Verfall vor. Ein Modellprojekt in Baden-Württemberg hat hier gezeigt, dass es sich beim Verfall nach $\S 73$ StGB um ein sehr wirksames Mittel handeln kann, den Drahtziehern des Menschenhandels die wirtschaftliche Grundlage zu entziehen. Es konnte sehr effizient verhindert werden, dass nach einer Verhaftung des Täters und einer Schließung des Bordells sofort einige Wochen später Etablissements durch Nachfolger eröffnet wurden. Hier hat man sich über die vom $\mathrm{BGH}^{10}$ erneut bestätigte rechtliche Hürde des § 73 StGB hinweggesetzt, wonach nur solche Vermögenswerte entzogen werden dürfen, die der Täter aus der illegalen Transaktion erlangt hat. Ferner nur solche, die er benötigt, um Schadensersatzansprüche zu begleichen. Werden die Frauen nach einer Razzia vernommen oder nach ihrer Aussage abgeschoben, können sie diese Schadensersatzansprüche nicht mehr geltend machen, aber dem Täter bleibt ein Restvermögen in erheblicher Höhe. Um den erweiterten Verfall bundesweit konsequent anzuwenden, müsste $§ 73$ StGB auf das gesamte Vermögen des Täters ausgedehnt werden, d.h. der Täter dürfte auch nichts zurückbehalten um die Schadensersatzansprüche zu begleichen ${ }^{11}$. Ein tatsächlicher Ausgleich müsste für die Frauen über die Staatskassen - und das eingezogene Tätervermögen - erfolgen. Ein weiterer Vorteil dieses Verfahrens wäre der schnellere Zugriff auf das Vermögen der Täter, sie hätten in der Praxis weniger Zeit ihr Vermögen beiseite zu schaffen.

\section{Aufenthaltsrechtlicher Status und Zeugenschutzmaßnahmen}

Im Rahmen der Verhandlungen zum Zuwanderungsgesetz ${ }^{12}$ wurden die Fälle des Frauenhandels nicht mitdiskutiert. Ein Versäumnis, denn ein Ausgleich der systematischen Wertungswidersprüche zwischen ausländer-, straf- und zeugenschutzrechtlichen Bestimmungen ist dringend erforderlich. Er kann nur noch auf der Verordnungsebene erfolgen, was konkret bedeutet, dass auch hier Ermessensspielräume bestehen, die es weiter erschweren für die betroffenen Frauen einen sicheren Aufenthaltstitel zu erlangen und sie vor Abschiebungen zu schützen. ${ }^{13}$ Wie hat sich die Situation der betroffenen Frauen nun durch den EU-Beitritt und das Zuwanderungsgesetz verändert? Für die Frauen aus den neuen Beitrittsländern ${ }^{14}$ hat sich die Situation verbessert. Sie können frei ins Bundesgebiet einreisen und sind daher wegen illegalen Aufenthaltes, auch nicht von Abschiebungen bedroht. Einziger Unterschied zu anderen EU-BürgerInnen: es gilt noch nicht die volle Arbeitnehmerfreizügigkeit, d.h. sie besitzen lediglich die Dienstleistungs- und noch nicht die Niederlassungsfreiheit. ${ }^{15}$ Konkret können die einreisenden Frauen aber bereits jetzt selbständige Tätigkeiten ausüben, die nicht von einer Erlaubnis der Arbeitsagentur abhängen. ${ }^{16}$ Auch wenn sie sich als Touristinnen oder Saisonarbeiterinnen hier aufhalten, können sie ein selbständiges Gewerbe anmelden oder zur Ausübung dieser Tätigkeit in Deutschland bleiben. ${ }^{17}$ Anders sieht es bei den Frauen aus Nicht EU Ländern aus. Sie benötigen nach wie vor ein Visum, das nur für 3 Monate gilt und bis auf Saisonarbeiten, keine Beschäftigung darüber hinaus erlaubt. Eine Duldung nach diesen drei Monaten darf nur noch erteilt werden, wenn ein rechtliches oder tatsächliches Abschiebehindernis nach § 60a Abs. 2 AuslG besteht oder das Landesinnenministerium die Abschiebung nach $\S 60$ a Abs. 1 AuslG für maximal 6 Monate aussetzt. Für längere Zeiträume kann keine Duldung mehr erteilt werden, sondern nur eine Aufenthaltserlaubnis, für die eine Abstimmung mit dem Bundesinnenminister nötig ist. Die bisher in vielen Fällen eingesetzten Verlängerungsduldungen nach § $55 \mathrm{Abs}$. 3 AuslG fallen künftig weg, der Aufenthalt muß beendet werden wenn. Er kann nur noch über eine Festsetzung der Ausreisefrist auf maximal 6 Monate hinausgezögert werden. Bestehen Anhaltspunkte für einen Menschenhandel darf nicht sofort, sondern frühestens nach vier Wo- chen eine Abschiebung erfolgen, d.h. innerhalb dieser Frist ist die Beweislage zu prüfen. ${ }^{18} \mathrm{Im}$ Umkehrschluss würde dies bedeuten, dass vor einer Abschiebung immer Anhaltspunkte für einen Menschenhandel zu prüfen wären. Werden sie bejaht, ist eine Verlängerung des Aufenthalts aus humanitären Gründen nach $\S 25$ Abs. 4 AuslG iVm Nr. 8 der Kooperationsrichtline für Menschenhandelsopfer möglich ${ }^{19}$, d.h. sie müssen sich als Zeuginnen zur Verfügung stellen, ansonsten droht gleich die Abschiebung. Ohne Zeugenschutzmaßnahmen sind aber nur wenige Frauen zur Aussage bereit und es bleibt für sie nur die Abschiebung, sie können keinen Asylantrag stellen. Nach § 15 a AuslG - neu sollen die Frauen bis zur Klärung der Aufenthaltsfragen und des Verfahrens (Abschiebung oder Zeugenaussage im Verfahren) in einem ersten Schritt künftig dem Bundesamt für Flüchtlinge zugeteilt und nach Kontingenten den einzelnen Bundesländern zugewiesen werden. Eine willkürliche Zuteilung, die die psychosoziale Begleitung und Betreuung der Frauen durch Opferhilfseinrichtungen erschwert, denn die erste Anlaufstelle im polizeilichen "Aufgriffsort« der Frauen hat meist keine entsprechenden Partnerstellen in den Zuteilungsorten und in jedem Fall muss eine neue persönliche und fachliche Betreuung organisiert werden. Hier müsste durch Verordnungen geregelt werden, dass die Frauen im Rahmen der Zuteilung gleich durch entsprechende Fachberatungsstellen beraten werden, oder - wenn Aufnahmekapazitäten bestehen dort aufgenommen werden können. Hinzu kommen die praktischen Schwierigkeiten der sozialen Absicherung der Frauen, denn die Sozialämter verweigern meist die Zahlung, da kein gesicherter Aufenthaltstitel besteht und sie sich für unzuständig erklären. Hier müssten ebenfalls über den Weg von Verordnungen entsprechende Zuständigkeiten geklärt werden. Eine weitere Hürde ist dann der Aufenthaltstitel, denn auch durch die skizzierte Kooperationsrichtline ${ }^{20}$ erhalten die Frauen einen - befristeten und zweckgebundenen Aufenthaltstitel. Welcher Zweck und welche Frist, das bleibt unklar. Damit gilt die bisherige Praxis, dass nur im Fall der Aussage der Frauen eine Aufenthaltsoption - zumindest für die Dauer des Verfahrens besteht. Was aber wenn die Frauen nicht aussagen wollen, oder gar nicht wegen Frauenhandels ermittelt wird? Hier bleibt - ausländerrechtlich - nur die Abschiebung der Frauen.

\section{Einstellungsvorschrift des § 154c StPO}

Die Staatsanwaltschaft kann künftig nach § 154c $\mathrm{StPO}^{21}$ von einer Verfolgung wegen eines illegalen Aufenthaltes oder strafrechtlichen Vergehen der Frauen absehen. Die Regelung wird dem tatsächlichen Bedürfnis der Opfer nach Sicherheit vor eigener Strafverfolgung (wegen marginaler Vergehen, gemessen an dem anzuzeigenden Menschenhandel, insbesondere in der qua- 
lifizierten Form des Verbrechens) nicht gerecht. Problematisch ist auch, dass das die Frauen eigene Straftaten schon bei der Anzeige der gegen sie verübten Taten oder in ihren Vernehmungen als Zeugen offenbaren müssen. Die Zusicherung, dass sie selbst nicht verfolgt werden, kann ihnen nicht - wie es für eine effiziente Ermittlung des Menschenhandels nötig wäre - vorab gegeben werden, weil die Ermessensbetätigung der Staatsanwaltschaft von der Kenntnis dieser zu offenbarenden Straftat abhängt. Zusätzlich wird eine großzügige und sachgerechte Anwendung des $\S 154 \mathrm{c}$ StPO dadurch erschwert, dass die Richtlinien für das Straf- und Bußgeldverfahren die Entscheidung über die Zusicherung der Nichtverfolgung dem Behördenleiter vorbehalten. Die Regelung soll die Aussagebereitschaft fördern. Es ist aber absehbar, dass es in der Praxis nicht ausreicht Straffreiheit zuzusichern, solange die Frauen noch Angst vor persönlicher Verfolgung durch die Täter haben. Wichtig wären daher entsprechende (Zeugen-) Schutzmaßnahmen für die Frauen. Derzeit werden weniger als $2 \%$ der Opferzeuginnen in entsprechende polizeiliche Zeugenschutzprogramme aufgenommen, da erstens die rechtlichen Hürden dafür zu hoch sind ${ }^{22}$ und zweitens ihre Handhabung für die Frauen zu unflexibel und wenig praktikabel sind. Entsprechende neue Kooperationskonzepte zwischen Polizei und Fachberatungsstellen, wie sie im Arbeitskreis Frauenhandel auf Bundesebene erarbeitet wurden, werden derzeit vor Ort auf Länderebene noch nicht ausreichend umgesetzt. Skizzierte Kooperationsrichtlinie verweist darauf, dass Menschenhandelsopfern die mit den zuständigen Behörden kooperieren entgegenzukommen ist, dies umfasst nicht nur ausländerrechtliche, sondern auch zeugenschutzrechtliche Maßnahmen.

\section{Förderungshandlungen}

Nach der Anhörung von ExpertInnen wurde im Vermittlungsverfahren noch die Strafbarkeit von Förderungshandlungen aufgenommen (§ 233 a StGB). Es hat sich in der bisherigen Praxis gezeigt, dass ein anwerben, befördern, weitergeben und beherbergen einer Person im Einzelfall eben nicht unter die Systematik der Mittäterschaft oder Beihilfe zum Bringen in die Prostitution oder den sonst genannten Zwecken fällt. Die (Haupt-)Tat des Menschenhandels besteht in der Einflussnahme auf den Willen- und die Entschließungsfreiheit einer Person, die eben gerade durch diese Tathandlungen gefördert wird und nicht erst auf der Stufe der tatsächlichen sexuellen Ausbeutung. Ferner besteht nun die Option auch effizienter im erweiterten Täterumfeld und innerhalb der organisierten Strukturen zu ermitteln und nicht länger nur die Frauen als Zeuginnen in den Mittelpunkt des Verfahrens zu stellen, da sich das gesamte Ermittlungsfeld nun erweitern muss.

\section{Heiratshandel: Nötigung zur Eingehung einer Ehe}

Die derzeitige Regelung zum Heiratshandel ist entscheidend von der Baden-Württembergischen Vorlage abgewichen und wurde lediglich ergänzend im Rahmen der Nötigung geregelt. ${ }^{23}$ Der Entwurf sah demgegenüber einen $\S 234 \mathrm{~b}$ StGB vor, der sich an die bisherigen Tatbestände der Nötigung, des Menschenhandels und der Verschleppung anlehnt. Im Zivilrecht soll die Aufhebung einer durch widerrechtliche Drohung zustande gekommenen Ehe durch den Wegfall der Antragsfrist von einem Jahr erleichtert werden. Ferner sollte $\S 1318$ Abs. 2 Satz 1 Nr. 1 BGB dahingehend geändert werden, dass Unterhaltsansprüche des genötigten Ehegatten nicht mehr davon abhängen, dass die Drohung durch den anderen Ehegatten oder mit dessen Wissen vorgenommen worden ist. Damit sollte verhindert werden, dass der genötigte Ehegatte nur deshalb vom Aufhebungsvertrag absieht und das Scheidungsverfahren wählt, weil er sonst unterhaltsrechtlich Nachteile zu erwarten hätte. Schließlich sollte $§ 1318$ Ab s. 5 BGB für den Fall des Zustandekommens der Ehe durch widerrechtliche Drohung ergänzt werden: Beim Tod des genötigten Ehegatten soll das gesetzliche Erbrecht des anderen Ehegatten auch dann ausgeschlossen sein, wenn noch kein Antrag auf Aufhebung der Ehe rechtshängig ist. Die jetzige Regelung greift sehr kurz und regelt nur lapidar die Nötigungshandlung »Eingehung einer Ehe«. Die im Entwurf vorgeschlagenen Erweiterungen beziehen hingegen die familienrechtlichen Problemstellungen mit ein und stellen gute Lösungsansätze dar. Sie sollten erneut aufgegriffen werden.

\section{Weitere Forderungen aus Bayern}

Bayern hat am 18.3.2005 folgende Änderungen vorgeschlagen ${ }^{24}$ :

- Freier, die wissen, dass es sich um Zwangsprostituierte handelt, sollen strafrechtlich belangt werden

- Der Strafrahmen für das Verbringen von Kindern in die Prostitution von bisher einem bis zu zehn Jahren auf zwei bis zu 15 Jahren soll erhöht werden

- Die Telekommunikation bei allen Straftaten des Menschenhandels überwacht werden können

- Eine Kronzeugenregelung für Menschenhandelsdelikte eingeführt werden

- Das Prostitutionsgesetz wieder rückgängig gemacht und die Förderung der Prostitution wieder eingeführt, sowie der Tatbestand der Zuhälterei wieder erweitert werden.

Die Vorlage sieht in der »Nachfrage « eine Förderung der Zwangsprostitution und will systematisch die Förderhandlungen nach $§ 233$ a StGB auch auf diese Gruppe der Freier erstrecken.
Über Sinn und Unsinn der Freierbestrafung sind vielfältige Debatten geführt worden. Klar ist, die Nachfrage regelt das Angebot und solange es sich bei der Zwangsprostitution um ein Kontrolldelikt handelt und keine Anzeigepflicht für die Freier besteht, ändert sich der Kontrolldeliktscharakter nicht. Lediglich im Hinblick auf eine erleichterte Strafverfolgung der Täter, wird teilweise eine Freierbestrafung durch etwa eine Ausdehnung der Förderungsmerkmale oder eine wie auch immer ausgestaltete Anzeige- oder Garantenpflicht befürwortet. Hier ist aber Skepsis angebracht, denn die Erfahrungen aus anderen Ländern, insbesondere Schweden, zeigen, dass eine Freierbestrafung weder den betroffenen Frauen noch den Strafverfolgungsbehörden bei den Ermittlungen nützt, sondern eher die Situation der betroffenen Frauen verschlechtert und sie kriminalisiert.

\section{Resümeé}

Die systematische Umstellung weg von den Sexualdelikten hin zu den Delikten gegen die persönliche Freiheit ist ein wichtiger Schritt in die richtige Richtung, da es bei diesem Delikt um die Einflussnahme auf den Willen und die persönliche Freiheit der Frauen geht. Auch die Strafbarkeit der Förderungshandlungen eröffnet sicherlich gute Optionen im erweiterten Täterumfeld und den organisierten Strukturen zu ermitteln und entlastet daher die Frauen, als bisher meist einzige Zeuginnen. Allerdings weist die Neuregelung auch systematische Wertungswidersprüche auf, die sich vor allem bei der Auslegung des Merkmals »Ausbeutung « zeigen. Hier ist nicht auf wirtschaftliche sondern auf persönliche Ausbeutung abzustellen. Ferner fehlen auf der ausländer-, sozial- und zeugenschutzrechtlichen Ebene noch die entsprechenden Begleitmaßnahmen für eine effektive Strafverfolgung. Auch die von der Fachkommission zum Frauenhandel erarbeiteten Begleitkonzepte für die Frauen und entsprechende Präventionsmodelle sind umzusetzen. $\mathrm{Zu}$ kritisieren ist ferner die moralisierenden Debatte des Frauenhandels unter dem Banner der »Freierbestrafung « - die zwar zu recht vor negativen Konsequenzen der globalisierten Markstrukturen warnt, gleichzeitig aber deren Gesetzmäßigkeiten verkennt und die Nachteile auf Kosten der Frauen austrägt (Stichwort: Abschiebung, fehlender Zeugenschutz, keine Sozialleistungen). Die Lösung ist aus meiner Sicht auf der Ebene einer opferintegrativen Sicht auf die Strafverfolgung zu suchen, dazu zählt die Umsetzung von Zeugenschutzmaßnahmen und die Erleichterungen im Aufenthaltsstatus für Nicht EU-BürgerInnen sowie die Gewährung von Sozialleistungen während ihres Aufenthaltes und die Möglichkeit von Rückkehrhilfen und Ausbildungsprogrammen für die Frauen.

Die Verfasserin ist Fachhochschullehrerin in Koblenz 


\section{Literatur:}

BKA-Lagebild Menschenhandel, www.bka.de/lagebildmenschenhandel;

Heger, Martin, zum Einfluss des Prostitutionsgesetzes auf das Strafrecht, StV 03, 350, 356;

Koelger/Thoma/Welter-Katschup: Probleme der Strafverfolgung und des Zeuginnenschutzes in Menschenhandelsprozessen, eine Analyse von Gerichtsachten, 2001;

SOLWODI ( Hrsg.): Grenzüberschreitende Verbrechen-Grenzüberschreitende Zusammen-arbeit, Schutz, Beratung und Betreuung von Gewalt- und Menschenhandelsopfern, 2003

\section{Fußnoten:}

12003 wurden 431 Ermittlungsverfahren eingeleitet und 1254 Opfer registriert. Zur näheren Betrachtung der Gründe wurde ein Forschungsauftrag an das Max-Planck Institut in Freiburg vergeben, die Ergebnisse wurden für August 2004 angekündigt, liegen aber derzeit noch nicht vor.

2 Einige Auffälligkeiten kurz skizziert: die Anzahl der betroffenen Frauen aus Rumänien hat sich im Vergleich zum Vorjahr verdoppelt, die Fallzahlen aus Litauen sind rückläufig und aus Bulgarien nach wie vor konstant hoch. Den höchsten Anteil gemessen am Opferverhältnis zur Bevölkerungszahl hat Lettland.

3 Vorgaben der Vereinten Nationen (Zusatzprotokoll zur Verhütung, Bekämpfung und Bestrafung des Menschenhandels, insbesondere des Frauen- und Kinderhandels, zum Übereinkommen der Vereinten Nationen gegen die grenzüberschreitende organisierte Kriminalität) und der Europäischen Union (Rahmenbeschluss des Rates der Europäischen Union vom 19. Juli 2002 zur Bekämpfung des Menschenhandels $2002(2002 / 629)$ siehe dazu ABl. EG Nr. L 203 vom 1. August 2002 S. 1.

4 Ein weitergehender BundesratsE zur Freierbestrafung (BR-Drs. 738/4/04) wurde im Vermittlungsausschuss zwar abgelehnt, aber die $\mathrm{Zu}$ sage zur Einberufung eines ExpertInnengesprächs gemacht, das am 20.4. 2005 stattfinden soll. Bayern hat inzwischen erneut einen Antrag zur Freierbestrafung, erweitert um den Vorschlag einer Kronzeugenregelung am 19.3.2005 in den Bundesrat eingebracht, der in den Ausschüssen beraten wird, BR-Drs. 140/05 .

5 Begründung zur Vorlage BT-DRs. 15/4848,S.23. Hier wird für den Begriff der Ausbeutung explizit auf die Zuhälterei in § 180 Abs. 2 Nr. 2 StGB und § 291 StGB verwiesen, die sich an den Rahmenbeschluss zur Bekämpfung des Menschenhandels anlehnen (wirtschaftliche Ausbeutung und "vergleichbare Ausbeutungsverhältnisse«), ferner Begründung zur Vorlage BT-Drs. 15/3045.

6 Ursprünglich war hier eine Absenkung auf 18 Jahre vorgesehen, Vorlage BT-Drs. 15/3045.

7 BT-DRs. 15/4848, S. 23.

8 BGHR StGB $\S 180$ a Abs. 2 Nr. 2 Ausbeuten 1 und BGHR StGB § 181 a Abs. 1 Nr. 1 Ausbeuten 3. Danach liegt Ausbeutung vor, wenn ein planmäßiges und eigensüchtiges Ausnutzen der Prostitutionsausübung als Erwerbsquelle, zu einer spürbaren Verschlechterung der wirtschaftlichen Lage der Prostituierten führt.

9 BGH NStZ 1989, 67 ff. [Anm. der Red.: Die Entscheidung betraf $\$$ \& 181 a I Nr. 1, \& 180a I Nr. 2 a.F. StGB].
10 BGH 4 STR 186/04 - Beschluß vom 5. August 2004 ( LG Münster), Höchstrichterliche Rechtsprechung Strafrecht (HRRS) 2004 Nr. 834.

11 Fraglich ist aber, ob eine so grob berechnete Vermögensstrafe verfassungsgemäß ist (die Redaktion).

12 Gesetz über den Aufenthalt, die Erwerbstätigkeit und die Integration von Ausländern im Bundesgebiet (Aufenthaltsgesetz - AufenthG), erkündet als Artikel I des Gesetzes zur Steuerung und Begrenzung der Zuwanderung und zur Regelung des Aufenthalts und der Integration von Unionsbürgern und Ausländern $\mathrm{Zu}$ wanderungsgesetz, BGBl. 2004 I, Seite 1950). Zur Entwicklung vergleiche die am 1.7. 2004 vom Bundestag bestätigten Änderungen des Zuwanderungsgesetzes in BT-Drs. 15/2479 vom 1.7. 04. auch BR-Drs. 662/04 vom 3.9. 2004 Gesetzentwurf zur Änderung des Aufenthaltsgesetzes und weiterer Gesetze - dient der Abstimmung des Zuwanderungsgesetzes, des Hartz IV Gesetzes und weiterer Gesetze aufeinander und der Korrektur fehlerhafter Querverweise. Redaktionell angepasst werden soll u.a. das AufenthaltsG, das SGB II, das AZRG, das AsylbLG, das AsylVfG und das SchwarzarbeitsbekämpfungsG. Zudem soll eine neue Datei zur Erfassung biomentrischer Daten von Ausländern ohne gültigen Pass eingeführt werden.

13 dazu die Umsetzungshinweise des Bundesinnenministeriums vom Dezember 2004 http://www.fluechtlingsinfo-berlin.de/fr/ gesetzgebung/BMI Hinweise AufenthG 221204.pdf).

14 Polen, Ungarn, Slowenien, Tschechische Republik, Slowakische Republik, Litauen, Lettland, Estland, Malta und Zypern. Die meisten Frauen kommen nach dem Lagebericht Menschenhandel aus der Türkei $(10,2 \%)$ und Bulgarien ( $8,3 \%)$, d.h. aus Nicht EU-Staaten. Für die nächste Gruppe ( Litauen, 8,2 \%) hat die Gültigkeit der EU-Regelungen hingegen Verbesserungen gebracht, sie können nicht wegen illegalen Aufenthalts abgeschoben werden.

15 Die Arbeitnehmerfreizügigkeit tritt erst nach einer Frist in Kraft. Die Regelung wird 2 plus 3 plus 2 Regelung genannt, da die Freizügigkeit zunächst für zwei Jahre ausgesetzt ist, anschließend kann sie durch Mitteilung an die Europäische Kommission für weitere 3 Jahre ausgesetzt werden und durch begründete Mitteilung nochmals für 2 Jahre. Einige EU-Staaten (Niederlande, Großbritannien) haben die Arbeitnehmerfreizügigkeit für die Beitrittsstaaten sofort herstellt, in Deutschland ist sie ausgesetzt. Freizügigkeitsberechtigt sind von den neuen EU-Staaten lediglich Malta und Zypern.

16 Beschäftigungsverordnung.

17 Das Gewerbe darf allerdings nicht unter die Beschränkungen der Dienstleistungsfreiheit fallen (z.B Baugewerbe) Beschäftigungsverordnung. Sie können aber auch Prostitution als Gewerbe anmelden. Handhabung in den Bundesländern hierzu unterschiedlich, Fachgespräch und ExpertInnenanhörung Bündnis 90/Grünen im Bundestag vom 16.8.2005.

18 Punkt 50.2.2. der Richtlinie zur Umsetzung des EU-Richtlinie des Rates über die Erteilung kurzfristiger Aufenthaltstitel für die Opfer der Beihilfe zur illegalen Einwanderung und des Menschenhandels, die mit den zuständigen Behörden kooperieren, verabschiedet am 29.4.2004 (RL 2004/81/EG,ABl.L 261/19 v.6.8. 2004). Alle weiteren Gründe für einen humanitären Aufenthalt § 25 Abs. 4 S. 1 AuslG: Eheschließung oder Lebenspartnerschaft mit deutschen Staatsangehörigen, die einen Anspruch auf
Aufenthaltserlaubnis vermittelt (so auch 55.3.2.2. VwV AuslG), die Anerkennung eines eigenen Kindes durch einen deutschen Vater und damit Feststellung der deutschen Staatsangehörigkeit oder die Übertragung des Sorgerechts für ein deutsches Kind, fortgeschrittene Schwangerschaft (VG Berlin, Inf AuslR 1995, 415),vorübergehende Betreuung eines schwer erkrankten Familienangehörigen (55.2.3.3. VwV AuslG), Abschluss einer medizinischen oder psychotherapeutischen Behandlung (55.3.2.1. VwV AuslG), Abschluss einer Schuloder Berufsausbildung, nur wenn kurz vor Abschlussstadium, Regelung von Familien- oder Nachlassangelegenheiten, Führung von Prozessen, der die Anwesenheit erforderlich macht, z.B. ein Vaterschaftsfeststellungsverfahren oder ein Scheidungsverfahren (nur wenn die persönliche Anwesenheit aber nicht durch eine kurzfristige Betretenserlaubnis ermöglicht werden kann, ablehnend für die Vaterschaftsfeststellung VG Berlin, InfAuslR 1996,397, siehe auch OVG Hamburg, EZAR 045 Nr. 3) Bundesinnenministerium vom Dezember 2004.

19 EU-Richtlinie des Rates über die Erteilung kurzfristiger Aufenthaltstitel für die Opfer der Beihilfe zur illegalen Einwanderung und des Menschenhandels, die mit den zuständigen Behörden kooperieren, verabschiedet am 29.4.2004 (RL 2004/81/EG,ABl.L 261/19 v.6.8. 2004). Alle weiteren Gründe für einen humanitären Aufenthalt § 25 Abs. 4 S. 1 AuslG: Eheschliessung oder Lebenspartnerschaft mit deutschen Staatsangehörigen die einen Anspruch auf Aufenthaltserlaubnis vermittelt (so auch 55.3.2.2.VwV AuslG), die Anerkennung eines eigenen Kindes durch einen deutschen Vater und damit Feststellung der deutschen Staatsangehörigkeit oder die Übertragung des Sorgerechts für ein deutsches Kind, fortgeschrittene Schwangerschaft (VG Berlin, Inf AuslR 1995,415),vorübergehende Betreuung eines schwer erkrankten Familienangehörigen (55.2.3.3. VwV AuslG), Abschluss einer medizinischen oder psychotherapeutischen Behandlung (55.3.2.1. VwV AuslG),Abschluss einer Schul- oder Berufsausbildung, nur wenn kurz vor Abschlussstadium, Regelung von Familien- oder Nachlassangelegenheiten, Führung von Prozessen, der die Anwesenheit erforderlich macht, z.B. ein Vaterschaftsfeststellungsverfahren oder ein Scheidungsverfahren (nur wenn die persönliche Anwesenheit aber nicht durch eine kurzfristige Betretenserlaubnis ermöglicht werden kann, ablehnend für die Vaterschaftsfeststellung VG Berlin, Inf AuslR 1996,397, siehe auch OVG Hamburg, EZAR 045 Nr. 3).

20 EU-Richtlinie des Rates über die Erteilung kurzfristiger Aufenthaltstitel für die Opfer der Beihilfe zur illegalen Einwanderung und des Menschenhandels, die mit den zuständigen Behörden kooperieren, verabschiedet am 29.4.2004 (RL 2004/81/EG,ABl.L 261/19 v.6.8. 2004).

$21 \S 154$ c StPO - neu

Zeigt das Opfer einer Nötigung oder Erpressung (\$§ 240, § 253 des Strafgesetzbuches) diese an $(\S 158)$ und wird hierdurch bedingt ein vom Opfer begangenes Vergehen bekannt, so kann die Staatsanwaltschaft von der Verfolgung des Vergehens absehen, wenn nicht wegen der Schwere der Tat eine Sühne unerlässlich ist.

22 Gefordert wird nach § 2 ein »wesentlicher Beitrag zu den Ermittlungen «, Gesetz zum Schutz gefährdeter Zeugen.

23 BR-Drs. 767/04

24 BR-Drs. 140/05 vom 19.3.2005 